\title{
Roboter müssen verständlich und vorhersehbar werden
}

\author{
M. Mara
}

Online publiziert am 14. November 2019

(c) Springer-Verlag GmbH Austria, ein Teil von Springer Nature 2019

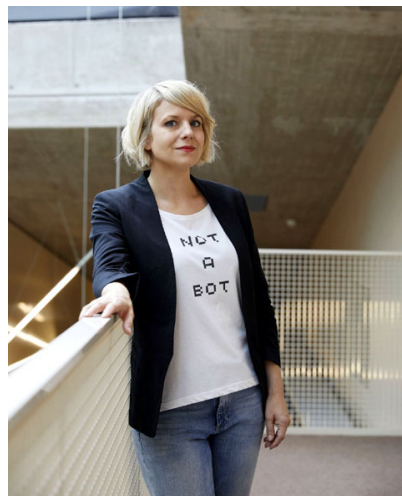

Wall-E, R2-D2 oder gar der Terminator. Staubsauger-Roboter, die über das Wohnzimmerparkett gleiten. Hochentwickelte Humanoide mit Silikonhaut und programmiertem Wimpernaufschlag. So genannte soziale Roboter, die medial als Pflegekraftersatz der Zukunft gehandelt werden. All das sind - mehr oder weniger fiktionale Bilder, die Menschen zum Begriff des "Roboters" in den Sinn kommen. Mit je-

Univ.-Prof.in Dr.in Martina Mara nen Roboterdomänen, die die International Federation of Robotics (IFR) in ihrem aktuellen Jahresreport als besonders relevant erachtet, korrespondiert dies freilich nur eingeschränkt. Wenig überraschend sind es dort nach wie vor industrielle Roboteranwendungen, die große Wachstumsraten und die stärkste Verbreitung in der Praxis aufweisen. Bis 2022 werden laut IFR global rund vier Millionen Industrieroboter im Einsatz sein.

Nachdem wir nun anstatt von Wall-E das Bild eines klassischen Industrieroboters im Kopf haben, jenes eines Manipulators zur Werkstückmontage beispielsweise: Spielt das Schwerpunktthema dieser Ausgabe - „Intelligente Roboter in Interaktion mit dem Menschen" - in diesem Bereich überhaupt eine Rolle? Sind Industrieroboter nicht üblicherweise hinter Absperrbändern oder gar in Käfigen untergebracht, um Interaktion mit Mitarbeiterinnen und Mitarbeitern aus Sicherheitsgründen zu vermeiden? Nach dem Motto: Sobald die Maschine läuft, haben Menschen dort nichts mehr verloren?

In vielen Fällen trifft das sicher noch zu. Ganz anders ist es allerdings im zunehmend populären Forschungs- und Entwicklungsfeld der Kollaborativen Robotik. Im Gegensatz zu herkömmlichen Industrierobotern sind kollaborative Roboter - kurz Cobots - nämlich leicht, sicher und intelligent genug, um körperlich nahe mit Menschen zusammenzuarbeiten. Cobots, die Seite an Seite mit Mitarbeitern etwa Verpackungen prüfen, Autositze montieren oder schweiBen, machen laut IFR drei Prozent des weltweiten Roboterumsatzes und damit ein Marktvolumen von rund 350 Millionen US-Dollar aus. Über Umsatzprognosen für die kommenden Jahre gibt es unterschiedliche Aussagen. Die allermeisten bewegen sich aber im Bereich zwischen fünf und 35 Milliarden US-Dollar bis zum Jahr 2025. Das lässt erwarten, dass Cobots und damit auch die physische Interaktion mit dem Menschen in immer mehr Arbeitsumgebungen Einzug halten werden. Der ehemals abgeschottete Industrieroboter wird somit zur sozialen Maschine.

Kommen sich Mensch und Maschine am Arbeitsplatz näher, tun sich neben der Leistungsfähigkeit eines Roboters plötzlich ganz neue
Fragen auf: Was braucht es, damit das Teamwork zwischen Mitarbeiter und Cobot funktioniert? Wie verständigen sich Mensch und Maschine am besten? Was macht ein angenehmes Kollaborationserlebnis aus, und wie können subjektive Sicherheit, Autonomie und Vertrauensbildung auf Seite des menschlichen Teamplayers gefördert werden? Wer Roboter baut, die neben der effizienten Erledigung ihrer Aufgaben auch Antworten auf diese Fragen geben, wird auf Dauer gewinnen. Gerade im Bereich von Cobots stellt die Mensch-Maschine-Schnittstelle ein marktentscheidendes Element dar.

Aktuelle empirische Forschungsarbeiten deuten darauf hin, dass Wohlbefinden und Sicherheitsempfinden in der Mensch-RoboterKollaboration unter anderem vom maschinellen Kommunikationsvermögen, von der "Lesbarkeit" des Roboters und damit zusammenhängend der Vorhersehbarkeit seiner Aktionen abhängt. Eine Prognose darüber ausbilden zu können, welches Ziel ein Kollaborationspartner verfolgt, ist - ganz generell - essenziell für gutes Teamwork. In der psychologischen Literatur zur kognitiven Vertrauensbildung stellen die Vorhersehbarkeit und das Einschätzenkönnen des Gegenübers wichtige Grundlagen für die Zuschreibung von Vertrauenswürdigkeit dar.

Für die Zusammenarbeit mit Cobots bedeutet das: So wie für den Roboter Zustände und Intentionen seines menschlichen Partners erkennbar sein müssen, müssen umgekehrt auch Zustände und geplante Aktionen des Roboterkollegen gut verständlich für den Menschen sein. In der Gestaltung dementsprechender Schnittstellen und Kommunikationssignale geht es dabei unter anderem um folgende Aspekte: Woran ist für den Menschen erkennbar, welches Objekt ein Roboter als nächstes greifen wird? Wie kommuniziert die Maschine, dass sie auf Input durch den Teampartner wartet? Durch welche Signale lässt sich für eine Person am besten einschätzen, in welche Richtung sich ein Roboterarm gleich bewegen wird?

Wie eine möglichst hohe Vorhersehbarkeit robotischer Aktionen in der Praxis umgesetzt wird, daran wird in den kommenden Jahren noch intensiv geforscht werden müssen. Erste Versuche weisen etwa am Roboter angebrachte Lichtsignale oder Projektionsmodule als vielversprechende Kommunikationswerkzeuge aus. Daneben könnte aber auch die Art, wie sich ein Roboterarm bewegt, einen großen Unterschied in punkto Verständlichkeit und Nutzerfreundlichkeit machen. Ein Beispiel: In einem Experiment von Anca D. Dragan und Kollegen von der Carnegie Mellon University wurde eine Coffeeshop-Situation nachgestellt, in der ein Roboter gemeinsam mit den Versuchsteilnehmern Getränkebestellungen abarbeitete. Der Roboter reichte seinen Partnern dabei in unbekannter Abfolge verschiedenfärbige Trinkgefäße. Diese mussten daraufhin die jeweils passende Zutat hinzufügen, zum Beispiel einen Pfefferminzteebeutel, sobald ein grüner Becher gereicht wurde.

Mara, Martina, LIT Robopsychology Lab, Johannes Kepler Universität Linz, Linz Österreich (E-Mail: martina.mara@jku.at) 
Das Ergebnis: Mensch und Maschine waren gemeinsam nicht am schnellsten, wenn der Roboter den effizientesten - also linearen - Weg zum nächsten Becher wählte, sondern dann, wenn seine Greifbewegungen kurviger - sprich: nach Vorbild menschlicher Armbewegungen - programmiert waren. Obwohl der Roboter für sich gesehen dadurch langsamer wurde, konnten die menschlichen Teampartner auf diese Weise schneller einschätzen, wohin der Roboter greifen würde und dementsprechend früher mit ihrem Teil der Aufgabe starten. In puncto Schnelligkeit schnitten die MenschMaschine-Teams mit den auf "Lesbarkeit" optimierten Roboterbewegungen dadurch sogar besonders gut ab. Aber das war nicht alles: Gleichzeitig bewerteten die Versuchsteilnehmer den Roboter mit den kurvigen Armbewegungen auch als weitaus angenehmeren Kollaborationspartner.

Genau um diese Verbindung von Effizienz und Akzeptanz, von Funktionalität und Verständlichkeit, muss es in der Gestaltung künftiger Mensch-Roboter-Interaktionen gehen. Am Arbeitsplatz und darüber hinaus.

Hinweis des Verlags Der Verlag bleibt in Hinblick auf geografische Zuordnungen und Gebietsbezeichnungen in veröffentlichten Karten und Institutsadressen neutral. 